\title{
NUEVO REGISTRO DE RHACHOEPALPUS OLIVACEUS TOWNSEND, 1908 (DIPTERA: TACHINIDAE) PARA PUEBLA, MÉXICO
}

\author{
NEW RECORD OF RHACHOEPALPUS OLIVACEUS TOWNSEND, 1908 \\ (DIPTERA: TACHINIDAE) FROM PUEBLA, MEXICO
}

\author{
LORENA SÁNCHEZ-GARCÍA, ${ }^{1}$ BENJAMÍN BARRIOS-DÍAZ, ${ }^{1}$ GLORIA VÁZQUEZ-HUERTA, ${ }^{1}$ \\ José MÉNDEZ-GÓMEZ, ${ }^{1}$ DULCE HERNÁNDEZ ZETINA² y ARMANdo EQUIHUA-MARTÍNEZ ${ }^{3}$
}

\footnotetext{
${ }^{1}$ Programa de Ingeniería Agroforestal, Facultad de Ingeniería Agrohidráulica BUAP, Av. Universidad SN, Barrio de Benito Juárez, Tetela de Ocampo, Puebla. CP.73640. e-mail: lorena.sanchez.garcia@hotmail.com, benjamin. barrios@correo.buap.mx; gloria.vazquezh@live.com.mx; jmendezg55@hotmail.com

${ }^{2}$ Departmento de Zoología, Instituto de Biología, Apdo. Postal 70-153, 04510 México, D.F., México. e-mail: zetinaadh@gmail.com

${ }^{3}$ Entomología y Acarología. Campus Montecillo. Colegio de Postgraduados. e-mail: equihuaa@colpos.mx
}

Recibido: 16/02/2016; aceptado: 01/11/2016

Editor responsable: Pedro Reyes Castillo

Sánchez-García, L., Barrios-Díaz, B., Vázquez-Huerta, G., Méndez-Gómez, J., Zetina, D. H. y Equihua-Martínez, A. (2017) Nuevo registro de Rhachoepalpus olivaceus Townsned, 1908 (Diptera: Tachinidae) para Puebla, México. Acta Zoológica Mexicana (n.s.), 33(1), 113-115.

RESUMEN. Este es el primer registro de Rhachoepalpus olivaceus Townsned, 1908 para el estado de Puebla, México. El registro está basado en varios especímenes encontrados al norte de Puebla, municipio de Capuluaque, específicamente en Tetela de Ocampo. Los especímenes fueron determinados con claves taxonómicas y depositados en la Colección de Insectos del Colegio de Postgraduados, Montecillo, Mexico (CEAM).

La familia Tachinidae comprende una gran diversidad de especies, algunas son parasitoides en su etapa larval de varios insectos, mientras que los adultos son de vida libre (Stireman et al. 2006). Por esta razón varias especies pueden ser consideradas como posibles agentes de control biológico de diversas plagas y otras participan de manera dinámica en la polinización de plantas (Wood 1987).

El género Rhachoepalpus fue propuesto por Townsend en 1908 y es reportado para las regiones Neotropical y Neártica de acuerdo con O'Hara (2016). Wood \& Zumbado (2010) mencionan que este género comprende cerca de 20 especies, con un rango de distribución des-
Sánchez-García, L., Barrios-Díaz, B., Vázquez-Huerta, G., Méndez-Gómez, J., Zetina, D. H., \& Equihua-Martínez, A. (2017) New record of Rhachoepalpus olivaceus Townsned, 1908 (Diptera: Tachinidae) from Puebla, Mexico. Acta Zoológica Mexicana (n.s.), 33(1), 113-115.

ABSTRACT. This is the first record of Rhachoepalpus olivaceus Townsned, 1908 for the state of Puebla, Mexico. The record is based on several specimens found in northern Puebla, municipality of Capuluaque, specifically in Tetela de Ocampo. The specimens were determined with taxonomic keys and deposited in the Insect Collection of Colegio de Postgraduados, Montecillo, Mexico (CEAM).

de USA hasta Argentina en altitudes variables, como por ejemplo en los bosques de los Andes. O'Hara (2017) cita para la región Neártica a Rhachoepalpus andinus Townsend, 1914; Rhachoepalpus argenteus Townsend, 1914; Rhachoepalpus beatus Curran, 1947; Rhachoepalpus biornatus Curran, 1947; Rhachoepalpus blandus $\mathrm{Cu}-$ rran, 1947; Rhachoepalpus cinereus Townsend, 1914; Rhachoepalpus ethelius Curran, 1947; Rhachoepalpus flavitarsis (Macquart, 1843); Rhachoepalpus immaculatus (Macquart, 1846); Rhachoepalpus metallicus Curran, 1947; Rhachoepalpus nitidus Townsend, 1914; Rhachoepalpus notatus Curran, 1947; Rhachoepalpus nova 
Curran, 1947; Rhachoepalpus ochripes (van der Wulp, 1888); Rhachoepalpus pulvurulentus (Schiner, 1868); Rhachoepalpus quatuornotatus (Townsend, 1935); Rhachoepalpus triformis (Walker, 1853); Rhachoepalpus tucumanus Blanchard, 1941 y Rhachoepalpus olivaceus Townsend, 1908. Rhachoepalpus immaculatus se ha reportado como parasitoide de la familia Saturniidae en Costa Rica (Janzen \& Hallwachs 2008). Wood (1987) reporta a Rhachoepalpus sp. para Costa Rica (Instituto de Biodiversidad-InBio); Ecuador (Colección Nacional de Canadá-CNC) mientras que Guimarães (1971) la reporta para México. O’Hara (2017) menciona que esta especie nominal es posible sinónimo de la especie tipo Rhachoepalpus testaceus (van der Wulp), descrita de Costa Rica. Sin embargo, se pueden diferenciar ambas especies porque $R$. testaceus no presenta el segmento anal emarginado en la parte media del borde posterior, mientras que $R$. olivaceus si lo presenta (Townsend 1908).

Rhachoepalpus olivaceus Townsend, llega a medir de 18 a $20 \mathrm{~mm}$ de longitud y presenta una coloración verde olivo, el segundo segmento antenal con una seda gruesa cerca del borde terminal y en algunas ocasiones pueden ser pareadas las sedas, escutelo con menos de cuatro hileras de sedas gruesas. Solo en el macho se presenta una línea media dorsal sobre el abdomen ensanchándose en el segundo segmento y con varias sedas gruesas. Presentan en ambos sexos el tercer segmento abdominal más estrecho que los otros, el segmento anal suavemente emarginado en la parte media del borde posterior, alas hialinas, las uñas en la hembra son amarillas con las puntas negras (Townsend 1908).

O'Hara (2017) y Wood \& Zumbado (2010) mencionan la presencia de $R$. olivaceus en Arizona, USA (Sabrosky \& Arnaud 1965), mientras que Townsend (1908) la reporta para México: Chihuahua, Sierra Madre, Río Piedras Verdes, 7000 pies; holotipo macho, Museo Nacional de Historia Natural (MNHN), Zetina (com. pers. 2016) menciona que también $R$. olivaceus se encuentra presente para los estados: Estado de México, Ciudad de México, Michoacán y Oaxaca (Colección Nacional de Insectos, Instituto de Biología, UNAM-CNIN), con el presente registro se amplía los datos de distribución para México, que alberga al estado de Puebla, el cual presenta una superficie de $34.251 \mathrm{~km}^{2}$, la mayor parte del territorio poblano se encuentra en el Eje Neovolcánico Transversal con clima que va del frío de las altas montañas al cálido semidesierto de la Mixteca en donde se desarrollan ecosistemas de bosques de encino (INEGI 2015). Capuluaque, Tetela de Ocampo se ubica en la Sierra Norte; presenta un clima templado subhúmedo con lluvias en verano, presenta la mayor parte de su territorio cubierto de bosques, tanto de pino, como de asociaciones de pino-encino, destacando el pino colorado, lacia y ayacahuite; encino colorado, cesante y oyamel, se encuentra ubicada a $2236 \mathrm{msnm}$ con coordenadas geográficas de $19^{\circ} 45^{\prime} 40.62 \mathrm{~N}$ y $97^{\circ} 47^{\prime}$ $46 \mathrm{~W}$ (INEGI 2015). El material fue colectado por medio de Trampa Malaise, en el periodo de invierno (09/ XII/12013) y se mantuvo en alcohol hasta su montaje con alfiler entomológico. Posteriormente se determinaron con ayuda de las claves taxonómicas de Wood \& Zumbado (2010) y el trabajo de Townsend (1908). También se buscaron especímenes adicionales del estado de Puebla en la Colección Nacional de Insectos del Instituto de Biología, UNAM (CNIN). Los ejemplares de la trampa Malaise se encuentran montados en seco y alfiler dentro de la Colección de Insectos del Colegio de Postgraduados, Monteci1lo, Estado de México (CEAM).

Material Examinado. México: Puebla, Capuluaque, Tetela de Ocampo, 2178 msnm, 09/XII/12013, Col. Sánchez-García (20, CEAM); $19^{\circ}$ 4’21.3” N y 097²5'53.2, Trampa Malaise; Carr. Puebla-Orizaba, 22/VII/1978, Col. J. Butze $(1 q$, CNIN).

\section{LITERATURA CITADA}

Guimarães, J. H. 1971. Family Tachinidae (Larvaevoridae). Papavero, N. (editor). A Catalogue of the Diptera of the South of the United States. Departamento de Zoologia. Secretaria de Agricultura, Sao Pablo, 333 pp.

INEGI, Instituto Nacional de Estadística y Geografía. 2015. Consultado en www.inegi.org.mx (acceso noviembre 2015).

Janzen, D. H. \& Hallwachs, W. 2008. 2005. Dynamic database for an inventrory of the macrocatepillar fauna, and its food plants and parasitoids, of Area de Conservacion Guanacaste (ACG), northwestern Costa Rica. Consultado http://janzen.sas.upenn.edu (acceso octubre 2015).

O'Hara, J. E. 2016. World genera of the Tachinidae (Diptera) and their regional occurrence. Version 9. PDF document, 93 pp. Published on the Internet at http://www.nadsdiptera.org/Tach/Genera/generahom.htm (acceso junio 2016)

O'Hara, J. E. 2017. Homepage for Tachinidae Resources. Taxonomic \& Host Catalogue of the Tachinidae of America North of Mexico. Available in: http://www.nadsdiptera.org/Tach/Genera/ (accessed junio 2016).

Sabrosky, C.W. \& Arnaud, J. R. 1965. Tachinidae. In: Stone, A. et al. (Eds.) A Catalog of the Diptera of American North of Mexico, State Departament of America. Agriculture Handbook $1696 \mathrm{pp}$.

Stireman, J. O., O’Hara, J. E. \& Wood, D. M. 2006. Tachinidae: evolution, behavior, and ecology. Annual Review of Entomology, 51, 525-555. 
Townsend, C. H. T. 1908. The Taxonomy of the Muscoidean Flies. Including Descriptions of New Genera and Species. Smithsonian Miscellaneous Collections, 51, 1-617.

Wood, D. M. 1987. Tachinidae. pp. 1193-1269. In: McAlpine, J. F., Peterson, V. B., Shewell, G. E., Teskey, H. J., Vockeroth, J. R. \& Wood, D. M. (eds.). Manual of Nearctic Diptera. Agriculture
Canada Monograph, Vol. 2, No. 28.

Wood, D. M. \& Zumbado, M. A. 2010. Tachinidae (tachinid flies, parasitic flies), pp. 1343-1417. In: B. V. Brown, Borkent, A., Cumming, J. M., Wood, D. M, Woodley, N. E. \& Zumbado, M. A. (eds.). Manual of Central American Diptera. NRC Research Press, Ottawa, Vol. 2. 